\title{
WAVE PROPAGATION AND THERMODYNAMIC LOSSES IN PACKED-BED THERMAL RESERVOIRS FOR ENERGY STORAGE
}

\author{
Alexander White ${ }^{1}$, Joshua McTigue ${ }^{1}$, Christos Markides ${ }^{2}$ \\ 1 Cambridge University Engineering Department, ajw36@cam.ac.uk \\ 2 Department of Chemical Engineering and Chemical Technology, Imperial College London
}

\begin{abstract}
This paper presents a numerical and theoretical analysis of thermal wave propagation in packed bed thermal reservoirs for energy storage applications. In such reservoirs, the range of temperatures encountered is usually such that the solid storage medium will exhibit significant changes in specific heat capacity. This in turn results in non-linear wave propagation and may lead to the formation of shock-like thermal fronts. Such effects have an impact on the exergetic losses due to irreversible heat transfer, and should be taken into account during the design and optimisation of the reservoirs. In the present paper, the emphasis is on thermal losses due to irreversible heat transfer. Frictional (pressure) losses and heat leakage between the storage medium and the environment are also important but are not considered here. The implications of the results for storage material, and particle size are discussed briefly in the context of loss minimisation.
\end{abstract}

Keywords: energy storage, irreversible heat transfer, thermal reservoirs, exergetic loss.

\section{NOMENCLATURE}

\section{Abbreviations}

AA-CAES

PHES

PTES

\section{Symbols}

$b=h-T_{0} S$

$c_{s}, c_{p g}$

$D$

e

$G$

$h=e+p / \rho$

$L$

$\dot{m}$

$p$

$S_{v}$

St $=\alpha / G c_{p g}$

$t$

$T$

$U$

$z$
Advanced Adiabatic Compressed Air Energy Storage

Pumped Hydro Energy Storage

Pumped Thermal Energy Storage

reservoir cross-sectional area, $\mathrm{m}^{2}$

flow availability per unit mass, $\mathrm{J} \mathrm{kg}^{-1}$

solid, gas (isobaric) specific heat capacity, $\mathrm{J} \mathrm{K}^{-1} \mathrm{~kg}^{-1}$

diameter of reservoir, $m$

internal energy per unit mass, $\mathrm{J} \mathrm{kg}^{-1}$

mass flow per unit area, $\mathrm{kg} \mathrm{s}^{-1} \mathrm{~m}^{-2}$

enthalpy per unit mass, $\mathrm{J} \mathrm{kg}^{-1}$

length of reservoir, $m$

mass flow rate, $\mathrm{kg} \mathrm{s}^{-1}$

pressure, $\mathrm{N} \mathrm{m}^{-2}$

entropy per unit mass, $\mathrm{J} \mathrm{K}^{-1} \mathrm{~kg}^{-1}$

solid surface area to volume ratio, $\mathrm{m}^{-1}$

Stanton number

time, $\mathrm{s}$

temperature, $\mathrm{K}$

wave speed, $\mathrm{m} \mathrm{s}^{-1}$

axial coordinate, $\mathrm{m}$ 


$\begin{array}{ll}\alpha & \text { heat transfer coefficient, } \mathrm{W} \mathrm{m}^{-2} \mathrm{~K}^{-1} \\ \varepsilon & \text { packed-bed void ratio } \\ \ell & \text { length scale (see Eq. } 7 \text { ), } \mathrm{m} \\ \rho & \text { density, } \mathrm{kg} \mathrm{m}^{-3} \\ \tau & \text { time scale (see Eq. } 8 \text { ), } \mathrm{s} \\ \tau_{s} & \text { particle surface shear stress, } \mathrm{N} \mathrm{m}^{-2}\end{array}$

\section{Subscripts}

Other symbols are defined in the text. Where appropriate, lower case symbols refer to specific quantities and upper case symbols to the corresponding extensive property.

\section{INTRODUCTION}

Fluctuations in electricity demand combined with the increased use of renewable generation technologies, many of which are inherently uncontrollable and intermittent, mean that there is increasing need for electrical energy storage schemes [1]. The majority of such storage is currently provided by pumped hydro (PHES) schemes, but PHES has very high capital costs and obvious geographical constraints. Many new storage technologies are thus being proposed and, amongst these, at least two make use of thermal reservoirs for storing all or part of the energy.

In Advanced Adiabatic Compressed Air Energy Storage (AA-CAES), the thermal energy resulting from compression is extracted from the air prior to its storage in large, high-pressure caverns. This energy is stored in some form of thermal reservoir and is returned to the air prior to its expansion through turbines during discharge. In some AA-CAES concepts, the thermal energy is extracted via heat exchangers and transferred to a specialised thermal fluid that is then stored in tanks. This has the advantage that the tanks may be at ambient pressure, but the thermal fluids are expensive and their temperature range limited. In other schemes, the thermal energy is stored in a solid medium such as concrete blocks or pebbles maintained within a packed bed. Zunft et al. [2] suggest that, despite the need for pressurised stores, this method provides the best solution.

A more recent storage technology to emerge that also makes use of packed-bed thermal reservoirs is variously known as PHES (Pumped Heat Electricity Storage) [3], PTES (Pumped Thermal Energy Storage, [4, 5]) or, in France, SEPT (Stockage d’Electricité par Pompage Thermique [6]). Here it will be referred to as PTES for consistency with the authors' previous publications and to avoid confusion with pumped hydro schemes. Figure 1 shows a simplified layout of a PTES system, comprising a compressor, C, an expander, $\mathrm{E}$, and hot and cold thermal stores, HS and CS respectively. C and $\mathrm{E}$ are linked mechanically and are connected to a motor-generator. During charge (as shown) the system effectively operates as a reverse Joule (or Brayton) cycle heat pump, extracting heat from CS and delivering heat to HS. During discharge, the processes are reversed such that the device operates as a closed Joule cycle heat engine. A $T$-s diagram for the reversible form of the cycle is shown in the figure, using argon as the working fluid and with a pressure ratio of 10:1 (this corresponds roughly to the scheme proposed in Ref. [3]). For the real cycle, heat must be rejected at some stage to counter the effects of irreversibility, and this is achieved via the heat exchangers HX1 and HX2.

Compared to AA-CAES, one of the main advantages of PTES is that it does not require high pressure gas storage and so is not subject to the same geographical constraints. (Most of the proposed CAES systems exploit solution-mined salt caverns or similarly large natural caverns for storing the compressed air.) On the other hand, PTES has twice as many compressions and expansions, making the round-trip efficiency particularly susceptible to losses during these processes. Further details of how PTES operates, including cycle analyses, are given in Refs. [4-6].

\subsection{Description of the packed-bed reservoirs}

The present paper is concerned solely with packed-bed reservoirs that use a solid storage material and a gaseous working fluid (e.g., air or argon). Energy is stored in the form of 'sensible heat' resulting from an increase (or decrease) in temperature of the solid, rather than from any change of phase. Note that for a cold reservoir, such as CS in Fig. 1 , the temperature when charged, $T_{c}$ , is less than that when discharged, $T_{d}$. Such a reservoir can nonetheless store available energy in the sense that work may be extracted via a heat engine operating between $T_{0}$ (the ambient temperature) and $T_{c}$.

The reservoirs of interest here typically comprise a cylindrical pressure vessel containing the solid storage medium in the form of a packed bed of pebbles or gravel, or a uniform matrix of ceramic. The vessel is insulated both internally and externally and is arranged vertically to minimise buoyancy effects, with gas entering from the top for hot reservoirs (during charge) and from the 
bottom for cold ones. For electrical energy storage applications, the main practical thermodynamic issues are the storage density (in, for example, $\mathrm{MJ} \mathrm{m}^{-3}$ ) and the storage efficiency. The latter may be defined as the available energy retrieved from the reservoir during discharge as a fraction of that entering during charge. Losses in the available energy stem from heat leakage to or from the environment, frictional pressure drop through the packed bed and irreversibility associated with heat transfer between the gas and solid. In the present work, heat leakage is assumed negligible and the focus is instead upon heat transfer irreversibility. This is justified since, in principle, any degree of insulation can be achieved.

The optimum design of the reservoir geometry, and in particular the length-to-diameter ratio, $L / D$, depends on a number of factors, including (amongst others) the cost of the containment material and insulation, the maximum allowable stresses, and the operating pressures and temperatures. From a purely thermodynamic perspective, the best value of $L / D$ is determined by a trade-off between pressure losses (which increase rapidly with $L / D$ ) and gas-solid heat exchange losses (which tend to fall with $L / D)$. Although pressure losses are included in this work they are not discussed due to space constraints and because they have virtually no impact on heat transfer related losses.

\subsection{Previous work}

A comprehensive description of design and analysis techniques for regenerators and packed-bed thermal stores is given by Schmidt and Willmott [7], and some of the modelling presented here is based on that work. The literature relating to packedbeds is indeed quite extensive, covering chemical and nuclear applications (e.g., [8]) as well as thermal storage. However, there are few publications relating specifically to electrical energy storage where efficiency considerations necessitate a Second Law analysis. Desrues et al. [6] undertook a numerical simulation of thermal reservoirs but only as part of the overall modelling of their turbomachinery-based PTES system. White [9] conducted a combined numerical and analytic study of reservoirs typical of a PTES system with the aim of identifying trends in thermal losses and their dependence on operating temperatures, geometry and pebble size. Ref. [9] also gives a preliminary analysis of loss minimisation by varying the ratio $L / D$. The present paper is an extension of the work presented in [9] with particular emphasis on the effects of the temperature dependent heat capacity of the storage medium. This has more than a mere quantitative impact since the resulting temperature-dependent thermal wave speed gives rise to additional phenomena that are both scientifically interesting and of practical importance in terms of their impact upon thermodynamic losses and interpreting test data.

\section{ANALYSIS AND WAVE PROPAGATION}

\subsection{Storage density and storage materials}

Since the focus is upon the storage of energy that is to be converted back to (electrical) work, we consider stored available energy, rather than simply internal energy. Thus, for a storage material of density $\rho_{s}$ packed with a void fraction $\varepsilon$, the change in stored energy between the discharged and charged states, per unit volume, is given by,

$$
\rho_{A}=\rho_{s}(1-\varepsilon)\left\{\left(e_{c}-e_{d}\right)-T_{0}\left(s_{c}-s_{d}\right)\right\} .
$$

For most packed beds $\varepsilon$ will typically be $\sim 30 \%$ so the main factor influencing the storage density is the product of the solid's density and heat capacity, $\rho_{s} c_{s}$. This is shown in Fig. 2 as a function of temperature for a few potential storage media. It is notable that over the temperature ranges of interest for storage technologies (e.g., between states 2 and 3 in Fig. 1 for hot reservoirs, and states 4 and 1 for cold ones) values of this product vary considerably. For the materials considered, $c_{s}$ increases with temperature, exhibiting a trend more or less in accord with the Debye crystal model.

Table 1 shows storage densities derived from Eq. (1) and average specific heat capacities for the above materials at conditions corresponding to the hot and cold reservoirs described in Appendix B. (Note that the choice of operating conditions is based approximately on a PTES system with a pressure ratio of 10:1, as in fig. 1, and with hot and cold reservoir discharge temperatures that are close to ambient. With argon as the working fluid this gives charging temperatures of $-150^{\circ} \mathrm{C}$ for the cold reservoir and $500^{\circ} \mathrm{C}$ for the hot reservoir). For comparison it is worth noting that for PHES with a $500 \mathrm{~m}$ altitude drop the energy stored is about $5 \mathrm{MJ}$ per $\mathrm{m}^{3}$ of water, whereas the figure for CAES at 100 bar is $\sim 36 \mathrm{MJ}$ per $\mathrm{m}^{3}$ of compressed air [5]. Energy storage densities for thermal reservoirs are thus relatively high. 
Table 1: Energy storage density and average specific heat capacity for various materials. (Reservoir details are given in Appendix B.)

\begin{tabular}{||c||c|c||c|c|}
\hline \multicolumn{1}{|c||}{} & \multicolumn{2}{c||}{ HOT STORE, $\mathrm{HS}$} & \multicolumn{2}{c|}{ COLD STORE, CS } \\
\cline { 2 - 5 } & $\begin{array}{c}\rho_{A} \\
\mathrm{MJ} / \mathrm{m}^{3}\end{array}$ & $\begin{array}{c}\bar{c}_{s} \\
\mathrm{~J} / \mathrm{kg} \mathrm{K}\end{array}$ & $\begin{array}{c}\rho_{A} \\
\mathrm{MJ} / \mathrm{m}^{3}\end{array}$ & $\begin{array}{c}\bar{c}_{s} \\
\mathrm{~J} / \mathrm{kg} \mathrm{K}\end{array}$ \\
\hline \hline $\mathrm{Al}_{2} \mathrm{O}_{3}$ & 560 & 1070 & 95 & 540 \\
\hline $\mathrm{Fe}_{2} \mathrm{O}_{3}$ & 585 & 850 & 125 & 500 \\
\hline $\mathrm{Fe}_{3} \mathrm{O}_{4}$ & 600 & 860 & 140 & 520 \\
\hline $\mathrm{SiO}_{2}$ & 360 & 1020 & 75 & 570 \\
\hline $\mathrm{TiO}_{2}$ & 470 & 855 & 110 & 550 \\
\hline
\end{tabular}

Table 1 also shows that the storage density for a cold reservoir is notably lower, typically by a factor of $\sim 4$, but for a PTES system usually less of the available energy needs to be stored in this reservoir. For example, for the system of Fig. 1 the rate at which available energy is supplied to CS is roughly half that of HS and consequently CS needs to be about twice the size. (This accords with the average solid heat capacity's being roughly a factor of 2 lower in CS.)

\subsection{Governing equations}

Figure 3 shows a schematic view of the heat transfer between the gas and solid for a hot reservoir during charge. The internal thermal resistance within the particles is small for the particle sizes of interest here and heat transfer is therefore limited by surface effects (i.e., the Biot number is effectively zero). Likewise, conduction along the bed has only a minor effect and is also ignored [7]. These assumptions essentially lead to the well-known Schumann model of thermal reservoirs, the basic correctness of which is well documented (see for example, [7]). Referring to the infinitesimal control volume (dashed line) of Fig. 3, the heat transfer rate between the gas and solid is thus given by,

$$
\delta \dot{Q}=A \delta z(1-\varepsilon) S_{v} \alpha\left(T_{g}-T_{s}\right)
$$

where $A$ is the open-tube cross-sectional area, $\alpha$ is the heat transfer coefficient and $S_{v}$ is the surface-to-volume ratio of the particles. The mass continuity, momentum and (gas and solid) energy equations for the same control volume yield,

$$
\begin{gathered}
\varepsilon \frac{\partial \rho_{g}}{\partial t}+\frac{\partial G}{\partial z}=0 \\
\frac{\partial G}{\partial t}+\frac{\partial}{\partial z}\left(\frac{G^{2}}{\rho_{g}}+\varepsilon^{2}\left(p-\rho_{g} g z\right)\right)=-\varepsilon(1-\varepsilon) \tau_{s} S_{v} \\
\varepsilon \frac{\partial}{\partial t}\left(\rho_{g} e_{g}\right)+\frac{\partial}{\partial z}\left(G h_{g}\right)=(1-\varepsilon) S_{v} \alpha\left(T_{s}-T_{g}\right) \\
\rho_{s} c_{s} \frac{\partial T_{s}}{\partial t}=S_{v} \alpha\left(T_{g}-T_{s}\right)
\end{gathered}
$$

where $G=\dot{m} / A$ is the mass flow rate per unit (open-tube) cross-sectional area and $\tau_{s}$ is the effective shear stress on the particle. For accurate calculations Eqs. (3-6) should be solved in a coupled fashion using, for example, a finite-volume time-marching approach. However, this would require very short time steps and is in any case unnecessary since the unsteady gas terms in Eqs. (3-5) are generally very small. For example, in Eq. (3), the temporal changes in gas density arise principally from the progression of the thermal wave (shown on the right of Fig. 3) and this occurs very slowly. Neglecting these unsteady terms gives $G=$ const. and also allows the energy equations to be solved independently of the momentum equation. Treating the working fluid as a perfect gas, Eqs. (5 \& 6) thus become, 


$$
\begin{gathered}
\frac{\partial T_{g}}{\partial z}=\frac{T_{s}-T_{g}}{\ell}=-\frac{\Delta T_{g s}}{\ell} \\
\frac{\partial T_{s}}{\partial t}=\frac{T_{g}-T_{s}}{\tau}=\frac{\Delta T_{g s}}{\tau}
\end{gathered}
$$

where $\Delta T_{g s}$ is the gas-solid temperature difference, and $\ell$ and $\tau$ are length and time scales given by,

$$
\begin{gathered}
\ell=\frac{G c_{p g}}{\alpha(1-\varepsilon) S_{v}}=\frac{1}{S t(1-\varepsilon) S_{v}} \\
\tau=\frac{\rho_{s} c_{s}}{\alpha S_{v}}=\frac{\rho_{s} c_{s}}{G c_{p g} S_{v} S t}
\end{gathered}
$$

St is the Stanton number and is obtained from packed-bed correlations presented in Ref. [12].

Wave propagation based on the model equations (5a) and (6a) is investigated below but numerical results are also presented for comparison and for the purpose of evaluating

losses where an analytical solution is not tractable. The numerical scheme used, based on Eqs. (3-6), is outlined in Appendix A.

\subsection{Ideal hot reservoirs}

An ideal reservoir would be one for which there are no irreversibilities due to heat transfer or fluid friction, and for which the gas exits at temperature $T_{d}$ (during charge). The rate at which available energy is stored within such a reservoir is thus given by,

$$
\dot{B}_{i n}=\dot{m}\left(b_{c}-b_{d}\right)=\dot{m} c_{p g}\left\{T_{c}-T_{d}-T_{0} \ln \left(\frac{T_{c}}{T_{d}}\right)\right\} .
$$

As shown below, the temperature dependence of $c_{s}$ causes the thermal front in hot reservoirs to become progressively less steep

during charge. This means that heat transfer occurs over an increasing interfacial area and thus with a decreasing gas-solid temperature difference as the wave progresses. It is therefore possible, at least in theory, for the heat transfer to occur more or less reversibly. (The large interfacial surface required would of course incur high pressure losses, but these can be treated separately.) The progress of the thermal front in this thermally ideal case may be determined by first combining Eqs. (5a) and (6a) to give,

$$
\frac{\partial T_{s}}{\partial t}+U \frac{\partial T_{g}}{\partial z}=0
$$

where $U$ is the thermal wave speed,

$$
U=\frac{\ell}{\tau}=\frac{G c_{p g}}{\rho_{s} c_{s}(1-\varepsilon)}
$$

Writing $T_{g}=T_{s}+\Delta T_{g s}$ and substituting for $\Delta T_{g s}$ from Eq. (5a), Eq. (10) becomes,

$$
\frac{\partial T_{s}}{\partial t}+U \frac{\partial T_{s}}{\partial z}=\frac{\ell}{\tau} \frac{\partial}{\partial z}\left(\ell \frac{\partial T_{g}}{\partial z}\right) .
$$

In this form the combined gas-solid energy equation can be interpreted quite clearly as wave propagation at speed $U$ combined with a dissipative process for which the effective diffusivity is,

$$
D=\frac{\ell^{2}}{\tau}=U \ell=\frac{U}{\operatorname{St}(1-\varepsilon) S_{v}} .
$$

Thermodynamic losses associated with irreversible heat transfer will thus be reduced if the length scale $\ell$ is made as small as possible. This is of course achieved by using very small particles $\left(S_{v} \rightarrow \infty\right)$ or high Stanton numbers, both of which are at odds with reducing pressure losses. As $\ell \rightarrow 0$, the gas-solid temperature difference disappears and the progress of the thermal front is by pure convection, its profile changing solely due to the temperature-dependent wave speed, Eq. (11). Since, as shown in Fig. $2, c_{s}$ generally increases with temperature, the cold 'nose' of the front travels fastest, causing the profile to become less steep. This process is reversible and the front would steepen again were the reservoir to be discharged. The shape of the temperature wave at a time $t$ after the beginning of charge is found by simply progressing each part of the initially discontinuous profile a distance 
$z=U\left(T_{s}\right) t$. Figure 4 shows the resulting temperature profile for the hot store detailed in Appendix B. The variation of $c_{s}$ used for this case is a linear approximation to the data for $\mathrm{Fe}_{2} \mathrm{O}_{3}$ (haematite). Numerical solutions with particle diameters of 20 mm and $1 \mathrm{~mm}$ are also shown and indicate that the ideal (analytical) result is approached as $\ell \rightarrow 0$, as expected. Practical particle diameters for such a reservoir would be $10-20 \mathrm{~mm}$ in order to avoid excessive pressure loss, and in this range it is clear that the dissipative term in Eq. (10a) plays a significant role.

\subsection{Stored energy and entropy for ideal reservoirs}

It may at first seem curious that an ideal reservoir should adopt a non-uniform temperature profile whilst being heated by a stream of gas at fixed temperature. This is however consistent with the First and Second Laws for a reversible process as we now show.

Firstly, the energy stored within the solid at any state of charge relative to the fully discharged state is given by,

$$
\Delta E_{s}=\int_{0}^{M_{d}}\left(e_{s}-e_{d}\right) d M_{s}
$$

where $e_{s}$ is the solid internal energy at temperature $T_{s}, M_{s}$ is the cumulative mass of solid at or above temperature $T_{s}$, and $e_{d}$ and $M_{d}$ are corresponding values at temperature $T_{d}$. With reference to Fig. 5, this integral may be rewritten as,

$$
\begin{aligned}
\Delta E_{s} & =M_{c}\left(e_{c}-e_{d}\right)+\int_{M_{c}}^{M_{d}}\left(e_{s}-e_{d}\right) d M_{s} \\
& =M_{c} e_{c}-M_{d} e_{d}+\int_{T_{c}}^{T_{d}} e_{s} \frac{d M_{s}}{d T_{s}} d T_{s}=\int_{T_{d}}^{T_{c}} M_{s} c_{s} d T_{s}
\end{aligned}
$$

the last form being obtained by integrating by parts. At time $t$, the point on the thermal front at temperature $T_{s}$ has travelled a distance $U t$ so, making use of Eq. (11),

$$
M_{s}=\rho_{s}(1-\varepsilon) A U t=\frac{\dot{m} c_{p g}}{c_{s}} t
$$

Substituting for $M_{s}$ into the above expression for $\Delta E_{s}$ gives,

$$
\Delta E_{s}=t \int_{T_{d}}^{T_{c}} \dot{m} c_{p g} d T_{s}=\dot{m} c_{p g}\left(T_{c}-T_{d}\right) t=-\Delta H_{g} .
$$

A similar analysis for the change in entropy of the solid gives,

$$
\Delta S_{s}=\int_{T_{d}}^{T_{c}} M_{s} \frac{c_{s} d T_{s}}{T_{s}}=\dot{m} c_{p g} \ln \left(\frac{T_{c}}{T_{d}}\right) t=-\Delta S_{g} .
$$

Equations (14) and (15) thus show that the internal energy increase of the solid is equal to the enthalpy drop of the gas and that the net entropy change is zero, consistent with the globally adiabatic and reversible nature of the process.

\subsection{Thermal shockwaves in cold reservoirs}

For cold reservoirs during charge, the non-linear wave speed acts so as to steepen the thermal front. This effect is evident in Fig. 6, which shows numerical simulations starting from an initially linear temperature profile. The steep gradients generated by wave 'catch-up' mean that the dissipative term on the right hand side of Eq. (10a) remains finite and it is thus no longer possible for heat transfer to take place reversibly, even if $\ell \rightarrow 0$.

As the cold front progresses through the reservoir, the tendency for wave catch-up will eventually be balanced by the effects of dissipation in a fashion similar to that within aerodynamic shockwaves. A steady-state shock-like profile is thus established and the temperature distribution of the solid must then be governed by,

$$
\frac{\partial T_{s}}{\partial t}+\bar{U} \frac{\partial T_{s}}{\partial z}=0
$$

where $\bar{U}$ is now independent of $T_{s}$. Since the thermal front now moves without change of shape, its passage through the reservoir has the effect of changing the temperature of a length of reservoir (equal the wave's displacement) from $T_{d}$ to $T_{c}$. Applying the unsteady flow energy equation (again neglecting the small unsteady gas term) thus gives, 


$$
\bar{U}=\frac{\dot{m} c_{p g}\left(T_{c}-T_{d}\right)}{(1-\varepsilon) A \rho_{s}\left(e_{c}-e_{d}\right)}=\frac{G c_{p g}}{(1-\varepsilon) \rho_{s} \bar{c}_{s}}
$$

i.e., the wave speed is that which would be given by Eq. (11) with $c_{s}$ replaced by its average value over the range $T_{c}$ to $T_{d}$.

\subsection{Temperature profiles in thermal shockwaves}

As an additional check on the validity of the numerical method, it is useful to derive an analytical expression for the solid and gas temperature profiles within the shock-like features described above. This is achieved by first using Eq. (5a) to recast Eq. (10a) in the alternative form,

$$
\frac{\partial T_{s}}{\partial t}+U \frac{\partial T_{s}}{\partial z}=-U \frac{\partial}{\partial z}\left(\tau \frac{\partial T_{s}}{\partial t}\right) .
$$

The time derivatives can then be eliminated by substitution from Eq. (16) to give (after straightforward manipulation),

$$
\left(\bar{c}_{s}-c_{s}\right) \frac{\partial \theta}{\partial \xi}=\frac{\partial}{\partial \xi}\left(c_{s} \frac{\partial \theta}{\partial \xi}\right)
$$

where

$$
\theta=\frac{T_{s}-\bar{T}_{s}}{T_{d}-T_{c}} \quad \text { and } \quad \xi=\frac{z-\bar{z}}{\ell}
$$

The quantities with over-bars are defined as the values that correspond to the average heat capacity - i.e.,

$$
\bar{c}_{s}=c_{s}\left(\bar{T}_{s}\right) \text { and } \bar{z}=z\left(\overline{\bar{s}}_{s}\right) \text {. }
$$

In deriving Eq. (18), it has also been assumed that $\ell$ varies only gradually with $z$. It may be possible to integrate this equation, depending on the form of $c_{s}\left(T_{s}\right)$. For example, if the variation is linear then, as shown in Appendix $C$, the temperature profiles are given implicitly by,

$$
\begin{gathered}
\xi=\left(\frac{2 \bar{c}_{s}}{\Delta c_{s}}-1\right) \ln (1+2 \theta)-\left(\frac{2 \bar{c}_{s}}{\Delta c_{s}}+1\right) \ln (1-2 \theta) \\
\phi=\frac{T_{s}-T_{g}}{T_{d}-T_{c}}=\frac{\Delta c_{s}}{4 \bar{c}_{s}}\left(1-4 \theta^{2}\right),
\end{gathered}
$$

where $\Delta c_{s}=c_{d}-c_{c}$. Figure 7 shows profiles of the solid-gas temperature difference profile for a cold shock front, computed from Eqs. (19) and (20) using a linear fit of $c_{s}\left(T_{s}\right)$ to data for $\mathrm{Fe}_{2} \mathrm{O}_{3}$ at low temperature. Numerical solutions are also shown, starting from a fully discharged initial state. In contrast to Fig. 6, the thermal front therefore gets less steep as it progresses due to dissipative effects. This is evident in the profiles of $\Delta T_{s g}$, which become less pronounced with time. In the steady state the numerical solution agrees very well with the analytical result with the minor differences being due almost entirely to the slight variation of $\ell$ as a result of Reynolds number effects (i.e., due essentially to the temperature dependence of $\alpha$ ).

\section{THERMODYNAMIC LOSSES}

An issue of obvious practical importance for electrical energy storage applications is the efficiency with which the energy can be stored. As discussed in Ref. [9], in addition to heat leakage effects, sources of availability loss include: (i) thermodynamic losses due to irreversible heat transfer; (ii) exit losses due to hot (or cold) gas issuing from the reservoir as it approaches full charge; (iii) storage losses due to the levelling off of thermal gradients during the period between charge and discharge; and (iv) pressure losses due to fluid friction. We focus here on the heat transfer irreversibility and, in particular, how this is affected by the temperature dependence of $c_{s}$. Exit, storage and pressure losses, although straightforward, are best considered in the context of the whole system; exit losses, for example, depend very much on the presence of other system components such as heat exchangers.

\subsection{Thermal losses in cold shockwaves}

As mentioned above, the abrupt thermal waves described in Sections 2.5 and 2.6 are akin to aerodynamic shockwaves and, like such shockwaves, they incur a thermodynamic loss that is independent of the detailed dissipative processes. The entropy 
generation rate is easily determined by noting, as before, that displacement of the thermal front by a distance $\delta z$ has the equivalent effect of changing a mass of solid corresponding to length $\delta z$ from $T_{d}$ to $T_{c}$. Thus,

$$
\dot{S}_{i r r}=\dot{S}_{s}+\dot{S}_{g}=\bar{U}(1-\varepsilon) A \rho_{s}\left(s_{c}-s_{d}\right)+\dot{m} c_{p g} \ln \left(\frac{T_{d}}{T_{c}}\right) \text {. }
$$

Substituting for $\bar{U}$ from Eq. (17) and rearranging then gives,

$$
\dot{S}_{i r r}=\dot{m} c_{p g} \int_{T_{d}}^{T_{c}}\left(\frac{c_{s}}{\bar{c}_{s}}-1\right) \frac{d T_{s}}{T_{s}}
$$

This is expressed as a loss coefficient by normalising by the net rate at which available energy enters the reservoir, as given by Eq. (8), giving

$$
\zeta_{t}=\frac{T_{0} \dot{S}_{i r r}}{\dot{B}_{i n}}=\frac{T_{0} \int_{T_{d}}^{T_{c}}\left(\frac{c_{s}}{\bar{c}_{s}}-1\right) \frac{d T_{s}}{T_{s}}}{T_{c}-T_{d}-T_{0} \ln \left(T_{c} / T_{d}\right)} .
$$

Figure 8 shows the evolution of the thermal loss coefficient for a cold reservoir undergoing charge. Numerical solutions are obtained by integrating the local entropy generation rate over the length of the reservoir, i.e.,

$$
\dot{S}_{i r r}=\int\left(\frac{1}{T_{s}}-\frac{1}{T_{g}}\right) d \dot{Q}=(1-\varepsilon) A S_{v} \int_{0}^{L} \alpha \frac{(\Delta T)^{2}}{T_{s} T_{g}} d z .
$$

Losses have been computed for two particle sizes and for an initial reservoir state that is either fully discharged or has a linear profile of temperature, as in the calculations of Fig. 6. In all cases the numerical results converge upon the value given by Eq. (22), confirming that the dissipation rate is independent of the details of the dissipative process. For comparison, a calculation with constant $c_{s}$ (set at its average value) is also plotted in Fig. 8 and in this case the loss falls continuously because there is no wave catch-up effect. To emphasise the importance of the wave catch-up effect, it may be noted that the total thermal loss incurred in charging the cold reservoir from empty to $80 \%$ full charge would be $19.1 \%$ of the incoming available energy with $\mathrm{Fe}_{2} \mathrm{O}_{3}$ as the storage material, but only $6.9 \%$ with a (fictitious) constant heat capacity material with the same average heat capacity.

Values of the shock loss based on Eq. (22) are shown in Table 2 for a few different storage materials and for both cold and hot reservoir conditions. In the latter case, the values tabulated would be the loss rates attained during discharge (if the reservoir is sufficiently long or the particles sufficiently small). Loss rates for the hot reservoir conditions are much lower, mainly due to the smaller fractional change in solid heat capacity at the higher temperatures. For the cold reservoir, the loss rates are really quite substantial, typically being about $15 \%$ of the incoming availability. There are, however, two mitigating factors that mean this does not have such serious implications for the overall efficiency of the storage process. Firstly, as stated above, cold reservoirs store a lower fraction of the available energy for a PTES system (roughly one third of the total in this case), so the loss factor must be weighted accordingly. Secondly, it is unlikely that the reservoirs would be operated in a manner that enables the thermal shocks to develop or persist for a significant time. The normal mode of operation is likely to be periodic cycling and, as shown below, this incurs much lower losses and is less susceptible to wave catch-up effects. 
Table 2: Shock loss coefficients computed from Eq. (22) for various materials, and fractional changes in heat capacity.

\begin{tabular}{|l||c|c||c|c|}
\hline \multicolumn{1}{|c||}{} & \multicolumn{2}{c||}{ COLD RESERVOIR } & \multicolumn{2}{c|}{ HOT RESERVOIR } \\
\cline { 2 - 5 } & $\zeta_{t}$ & $\frac{\Delta c_{s}}{\bar{c}_{s}}$ & $\zeta_{t}$ & $\frac{\Delta c_{s}}{\bar{c}_{s}}$ \\
\hline \hline $\mathrm{Al}_{2} \mathrm{O}_{3}$ & 0.237 & 1.067 & 0.043 & 0.381 \\
\hline $\mathrm{Fe}_{2} \mathrm{O}_{3}$ & 0.173 & 0.783 & 0.044 & 0.390 \\
\hline $\mathrm{Fe}_{3} \mathrm{O}_{4}$ & 0.129 & $0.202^{*}$ & 0.058 & 0.504 \\
\hline $\mathrm{SiO}_{2}$ & 0.164 & 0.739 & 0.052 & 0.458 \\
\hline $\mathrm{TiO}_{2}$ & 0.160 & 0.722 & 0.030 & 0.264 \\
\hline
\end{tabular}

${ }^{*}$ Value not representative due to the spike in $c_{s}$ shown in Fig. 2

Finally in this section, it is worth noting the relatively low value of shock loss obtained for $\mathrm{Fe}_{3} \mathrm{O}_{4}$ in the cold reservoir. This is due to the higher average $c_{s}$ and smaller variation over the temperature range, with a small benefit also coming from the spike in $c_{s}$ shown in Fig. 2. According to Ref. [11], this spike stems from a magnetic effect, but it is very localised so it is important that it be properly resolved when computing $\zeta_{t}$; initial calculations based on the (rather sparse) JANAF data [10] gave a shock loss of zero.

\subsection{Thermal losses during cyclic operation}

For load-levelling applications, reservoirs are likely to be charged and discharged on a regular (e.g., daily) basis. In this mode of operation, temperature profiles eventually become identical from one cycle to the next. As discussed in Ref. [9], the shape of these profiles and the magnitude of thermal losses will depend on the duration of the charge and discharge periods compared to the 'nominal' charge time of the reservoir, defined by $t_{n}=L / \bar{U}$.

Figure 9 shows computed profiles in a cold reservoir for cases with equal charge and discharge periods $\left(t_{c}=t_{d}\right)$ and with $(a) t_{c}=$ $0.50 t_{n}$ and (b) $t_{c}=0.75 t_{n}$. The material is either $\mathrm{Fe}_{3} \mathrm{O}_{4}$ or $\mathrm{Fe}_{2} \mathrm{O}_{3}$. The temperature dependence of $c_{s}$ clearly manifests itself as a steepening of the profiles during charge, but this effect is reversed during discharge. (Profiles computed with constant $c_{s}$ are shown for comparison in case (b).) Consequently, the shock-like features described above are absent and the variable $c_{s}$ thus has a less significant impact on losses. Loss coefficients are plotted in Fig. 10 and are seen to increase with the ratio $t_{c} / t_{n}$, due to the steeper gradients, as observed in Fig. 9. (Steep gradients imply heat transfer occurs over a smaller interfacial area and thus with a higher $\Delta T_{s g}$, thereby incurring greater loss.) The average temperature gradients obtained with constant and variable $c_{s}$ are in fact roughly the same (see Fig. $9(\mathrm{~b})$ ), but the fluctuation of these gradients between charge and discharge in the variable $c_{s}$ case generates slightly higher losses because entropy generation rates vary approximately as the square of temperature differences. It is notable that the loss coefficients for $\mathrm{Fe}_{2} \mathrm{O}_{3}$ and $\mathrm{Fe}_{3} \mathrm{O}_{4}$ with an imposed constant $c_{5}$ are almost identical but in the real, variable $c_{s}$ case, losses are higher for $\mathrm{Fe}_{2} \mathrm{O}_{3}$ due the higher fractional change in $c_{s}$.

Under cyclic conditions, losses are no longer independent of the effective diffusion coefficient (Eq. 12) and, as shown in Ref. [9], vary roughly in proportion to the length scale $\ell$. The thermal loss can therefore be reduced by decreasing the particle size, but this will be at the expense of higher pressure losses and a compromise must be sought.

\section{DISCUSSION AND CONCLUSIONS}

Based on the results presented in Sections 2 and 3, the following points of discussion and conclusions arise:

(i) The temperature dependence of the solid specific heat capacity has a strong influence on the shape of temperature profiles. In the first instance this has important implications for the interpretation of measurements made on packed beds since such measurements would be used, for example, to infer heat transfer coefficients. In this respect, data for Nusselt numbers given in [12] derived from packed bed tests show significant spread, typically encompassing a factor of about 4 . This uncertainty has implications for the prediction of thermodynamic losses.

(ii) Wave catch-up in cold fronts may lead to the formation of shock-like features within which the relative catch-up effect is exactly balanced by dissipation. As with aerodynamic shockwaves, the entropy generation rate within these shocks is 
independent of the detailed dissipative processes (i.e., it does not depend on the thermal resistance between the gas and solid) and increases with the fractional change in the solid's heat capacity over the relevant temperature range. The loss rates are high, particularly in cold reservoirs where they typically correspond to about $15 \%$ of the available energy. For an energy storage system operating in steady cyclic modes, such shocks can probably be avoided, but they may nonetheless contribute to thermodynamic losses during initial transient phases. It is therefore desirable to use storage materials that have a small fractional change in heat capacity between the charge and discharge temperatures.

(iii) The impact of variable solid heat capacity for cyclic operation is more modest. Losses in this case are much lower than for 'single charge' due to the less abrupt thermal fronts. Changes in $c_{s}$ still result in higher losses, with the effect increasing with the fractional change in $c_{s}$. However, losses under cyclic operation are dependent on the gas-solid thermal resistance and so optimisation of the particle size and reservoir geometry is desirable, taking into account also the effects of pressure loss.

(iv) The main methods of mitigating the potentially large losses associated with wave catch-up are (a) to make use of storage materials with a small fractional variation in heat capacity and (b) to avoid operating the reservoirs in a mode that allows the shock-like features to form. As noted above, the latter essentially means avoiding cold reservoirs from approaching a fully discharged state and hot reservoirs from becoming fully charged. A full assessment of the impact of the catch-up effect thus requires consideration of the 'duty cycle' of the storage device.

\section{APPENDIX A: The numerical scheme}

The computational method used for the numerical results presented in this paper is similar to that described in Ref. [9] but with minor refinements to treat variable solid and gas properties and to include the mass continuity and momentum equations.

The thermal behaviour of the reservoirs is governed chiefly by the two energy equations, (5) and (6). The energy equation for the solid may be written exactly as (6a), but Eq. (5a) neglects the gas unsteady term. This may be corrected by adding to the right hand side of (5a) the term:

$$
F=\frac{\varepsilon}{G c_{p g}}\left(\frac{\partial p}{\partial t}-\rho_{g} c_{p g} \frac{\partial T_{g}}{\partial t}\right)
$$

This term is obtained without approximation by combining Eqs. (3) and (5), but it is very small except during the initial transients for the charge (or discharge) of a fully discharged (or charged) reservoir.

Figure 11 shows part of the regular computational grid at two time levels. At a particular stage in the calculation, temperatures are known at all nodes for time step $n-1$ and at nodes up to and including $i-1$ at time step $n$. The task is thus to obtain values at node $(i, n)$. This is achieved by integrating Eq. (5a) (including the unsteady term $F$ ) between nodes $(i-1, n)$ and $(i, n)$ whilst holding $T_{s}$ and $F$ constant at their average values, giving:

$$
T_{g, i}^{n}=\left(\bar{T}_{s}+\bar{F} \ell\right)\left(1-\exp \left\{\frac{-\Delta z}{\ell}\right\}\right)+T_{g, i-1}^{n} \exp \left\{\frac{-\Delta z}{\ell}\right\}
$$

Similarly, Eq. (6a) is integrated between nodes $(i, n-1)$ and $(i, n)$ whilst holding $T_{g}$ at its average value, giving:

$$
T_{s, i}^{n}=\bar{T}_{g}\left(1-\exp \left\{\frac{-\Delta t}{\tau}\right\}\right)+T_{s, i}^{n-1} \exp \left\{\frac{-\Delta t}{\tau}\right\}
$$

The advantage of this semi-analytical approach is that the stiffness is integrated out of the equations, allowing larger space and time steps to be taken than with a direct finite difference method. An implicit scheme for integrating (A2) and (A3) is obtained by setting the average temperatures as:

$$
\bar{T}_{s}=\frac{T_{s, i-1}^{n}+T_{s, i}^{n}}{2} \text { and } \quad \bar{T}_{g}=\frac{T_{g, i}^{n-1}+T_{g, i}^{n}}{2}
$$

but numerical experimentation shows that it is sufficient to set the average $F$ based on values at $(i-1, n-1)$ and $(i-1, n)$ where all quantities are already known. The temperatures are then updated in a straightforward manner that involves inverting a $2 \times 2$ matrix, as described in Ref. [9]. Generally, solutions are 'grid' independent for space and time steps less than $20 \%$ of $\ell$ and $\tau$ respectively.

Since gas velocities are very small (usually a few $\mathrm{cm} / \mathrm{s}$ ) the only significant terms in the momentum equation are the pressure gradient and the frictional term. A straightforward pressure loss is thus computed between each node, based on a packed-bed skin friction correlation taken from Ref. [13]. Finally, the mass flow per unit area, $G$, varies very slightly through the reservoir due 
to the change in the mass of stored gas brought about by the passage of the thermal front. Eq. (3) is thus implemented in the form:

$$
G_{i}^{n}=G_{i-1}^{n}+\varepsilon \frac{\Delta z}{\Delta t}\left(\rho_{g, i}^{n-1}-\rho_{g, i}^{n}\right)
$$

It is worth noting that although the refinements included via equations (A1) and (A4) have only a very minor impact on temperature profiles, they are required to get the correct overall exergetic balance for the reservoirs.

The numerical scheme described above has been validated against the 'single-blow' analytical solution first presented by Anzelius [14] for the case of constant gas and solid properties. Details of this validation are given in ref. [9].

\section{APPENDIX B: Reservoir details}

The hot and cold reservoir geometries and gas mass flow rates used here have been chosen arbitrarily, except in that they are consistent with those in Ref. [9]. The operating temperatures, however, correspond roughly to those of the PTES system described in Ref. [3].

Table 3: Details of hot and cold reservoirs

\begin{tabular}{|l||c|c|}
\hline & Hot Reservoir & Cold Reservoir \\
\hline \hline Diameter & $5 \mathrm{~m}$ & $5 \mathrm{~m}$ \\
\hline Length & $5 \mathrm{~m}$ & $5 \mathrm{~m}$ \\
\hline Working fluid & Argon & Argon \\
\hline Mass flow rate & $12.5 \mathrm{~kg} / \mathrm{s}$ & $12.5 \mathrm{~kg} / \mathrm{s}$ \\
\hline Operating pressure & $10 \mathrm{bar}$ & $1 \mathrm{bar}$ \\
\hline Particle diameter & variable & variable \\
\hline Void fraction & 0.33 & 0.33 \\
\hline Charge temperature & $500{ }^{\circ} \mathrm{C}$ & $-150^{\circ} \mathrm{C}$ \\
\hline Discharge temperature & $25^{\circ} \mathrm{C}$ & $25^{\circ} \mathrm{C}$ \\
\hline
\end{tabular}

\section{APPENDIX C: Derivation of Eqs. (19) and (20)}

The profiles of temperature and temperature difference through the shock-like phenomena of Section 2.5 can be obtained analytically for restricted forms of the function $c_{s}\left(T_{s}\right)$. For most materials, a linear variation provides a reasonable fit with the data and is sufficient as a means of testing the numerical scheme and highlighting the physical processes. We thus write,

$$
c_{s}=\bar{c}_{s}+\Delta c_{s} \theta \quad \text { where } \quad \Delta c_{s}=c_{d}-c_{c}
$$

Substituting into Eq. (17) and integrating once gives,

$$
\frac{1}{4}-\theta^{2}=2\left(\frac{\bar{c}_{s}}{\Delta c_{s}}+\theta\right) \frac{\partial \theta}{\partial \xi} .
$$

The constant of integration (1/4) has been obtained here by noting that the gradient becomes zero as $\xi \rightarrow \pm \infty$, i.e., as $\theta \rightarrow \pm 1 / 2$. Separating the variables in Eq. (C1), integrating again using partial fractions and substituting the boundary condition, $\theta=0$ at $\xi=$ 0 , gives Eq. (19).

Eq. (20) is derived by combining Eqs. (6a) and (16) to give,

$$
\frac{T_{s}-T_{g}}{\tau}=\bar{U} \frac{\partial T_{s}}{\partial z}
$$

which can then be recast in the dimensionless form, 


$$
\phi=\frac{T_{s}-T_{g}}{T_{d}-T_{c}}=\frac{\bar{U} \tau}{\ell} \frac{\partial}{\partial \xi}\left(\frac{T_{s}-\bar{T}}{T_{d}-T_{c}}\right)=\frac{c_{s}}{\bar{c}_{s}} \frac{\partial \theta}{\partial \xi} .
$$

Differentiation of Eq. (19) and substitution into Eq. (C3) finally gives the normalised temperature difference, Eq. (20).

\section{ACKNOWLEDGEMENT}

The work described in this paper was undertaken as part of a project funded by the UK Engineering and Physical Sciences Research Council (EPSRC Grant No. EP/J006246/1). The authors gratefully acknowledge this support.

\section{REFERENCES}

[1] MacKay DJC. Fluctuations and storage, in: Sustainable Energy without the Hot Air, UIT

[2] Zunft S, Jakiel C, Koller M, Bullough C. Adiabatic com-pressed air energy storage for grid integration of wind power. Sixth Int. Workshop on Large-Scale Integration of Wind Power and Transmission Networks, Delft. (2006)

[3] MacNaghten J, Howes J. Energy Storage, Int. Patent No. WO 2009/044139 A2. (2009)

[4] White AJ, Parks GT, Markides CN. Thermodynamic analysis of pumped thermal electricity storage. (in press, available on-line) Applied Thermal Engineering. (2012)

[5] White AJ. Electricity storage using a thermal storage scheme, $8^{\text {th }}$ Int. Conf. of Comp. Methods in Science and Engineering (ICCMSE), Kos, Greece. (2010).

[6] Desrues T, Ruer J, Marty P, Fourmigué J. A thermal energy storage process for large scale electric applications, Applied Thermal Engineering. 30, pp. 425-432. (2010)

[7] Willmott A. Dynamics of regenerative heat transfer. Taylor \& Francis. (2002)

[8] Reitsma F. The pebble bed modular reactor layout and Neutronics design of the equilibrium cycle. The physics of fuel cycles and advanced nuclear systems. Chicago. (2004)

[9] White AJ. Loss analysis of thermal reservoirs for electrical energy storage schemes. Applied Energy. 88, pp. 4150-4159. (2011)

[10] NIST-JANAF Thermochemical Tables, available on-line at http://kinetics.nist.gov/janaf/ (accessed Feb 2013).

[11] Westrum EF, Grønvold F. Magnetite $\left(\mathrm{Fe}_{3} \mathrm{O}_{4}\right)$ heat capacity and thermodynamic peoperties from 5 to $350 \mathrm{~K} \mathrm{~J}$. Chem. Thermo. 1, pp. 543-557. (1969)

[12] Wakao N, Kaguei S, Funazkri T. Effect of fluid dispersion coefficients on particle-to-fluid heat transfer coefficients in packed beds: correlation of Nusselt numbers. Chem Eng Sci 1979;34:325-36.

[13] Holdich RG. Fundamentals of particle technology. Midland Information Technology and Publishing. (2002)

[14] Anzelius A. Heating by means of percolating media. J. Mech Des 6:291-294. (1926)

\section{FIGURE CAPTIONS}

Figure 1: Simplified layout of a PTES system and $T-S$ diagram for the reversible cycle.

Figure 2: Heat capacity per unit volume for various materials. Data are taken from Ref. [10], except for $\mathrm{Fe}_{3} \mathrm{O}_{4}$ which are from Ref. [11].

Figure 3: Schematic view of heat transfer process and typical axial temperature profiles for a hot reservoir during charge.

Figure 4: Solid temperature profiles for a hot reservoir during charge (geometry and other details are given in Appendix B).

Figure 5: Sketch of the solid temperature profile for an ideal hot reservoir as a function of the cumulative mass at or above $T_{s}$.

Figure 6: Numerical computation of wave 'catch-up' for a cold reservoir during charge. Geometry and other details are given in Appendix B. The storage material is $\mathrm{Fe}_{2} \mathrm{O}_{3}$. 
Figure 7: Solid-gas temperature difference profiles within a cold 'shock': comparison of the analytical result (eqs. 19 \& 20) with the numerical solutions. Geometry and other details are given in Appendix B. The solid is $\mathrm{Fe}_{2} \mathrm{O}_{3}$ with a linear fit of $c_{s}$ vs. $T_{s}$.

Figure 8: Evolution of the instantaneous loss coefficient, $\zeta_{t}$, for a cold reservoir undergoing charge. Geometry and other details are given in Appendix B. The material is $\mathrm{Fe}_{2} \mathrm{O}_{3}$.

Figure 9: Temperature profiles in a cold reservoir under cyclic operation for two different charge periods, $t_{c}$. In each case profiles are shown 5\%,50\% and 95\% through the charge period. Charge and discharge periods are equal in each case.

Figure 10: Thermodynamic loss for $20 \mathrm{~mm}$ diameter particles in a cold reservoir undergoing cyclic operation.

Figure 11: A section of the computation grid: temperatures are known at nodes marked $\bullet$ and unknown at nodes marked 0 . 


\section{FIGURES}
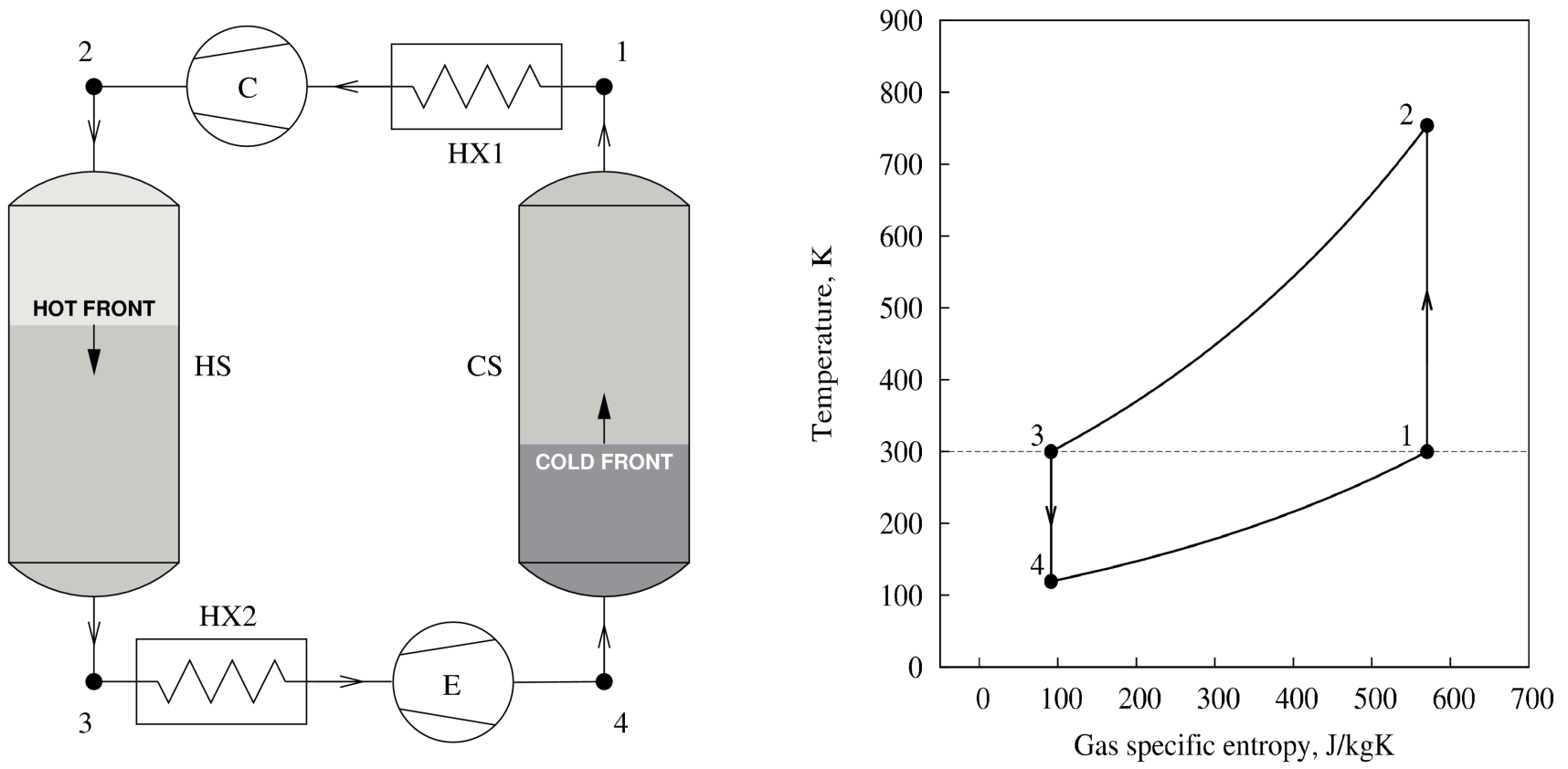

Figure 1: Simplified layout of a PTES system and $T-s$ diagram for the reversible cycle.

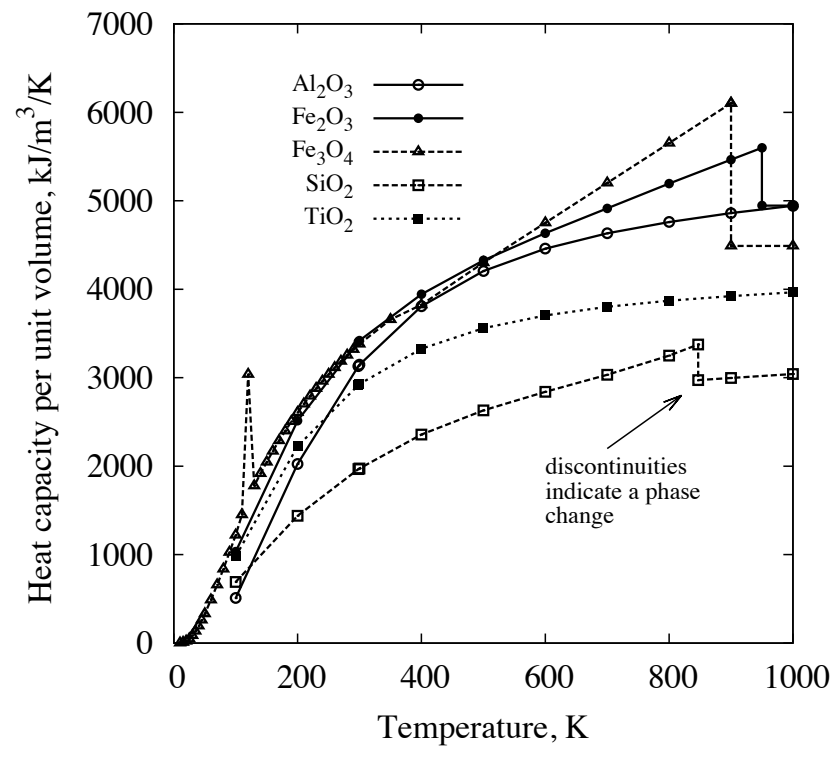

Figure 2: Heat capacity per unit volume for various materials. Data are taken from Ref. [10], except for Fe $\mathrm{O}_{4}$ which are from Ref. [11]. 

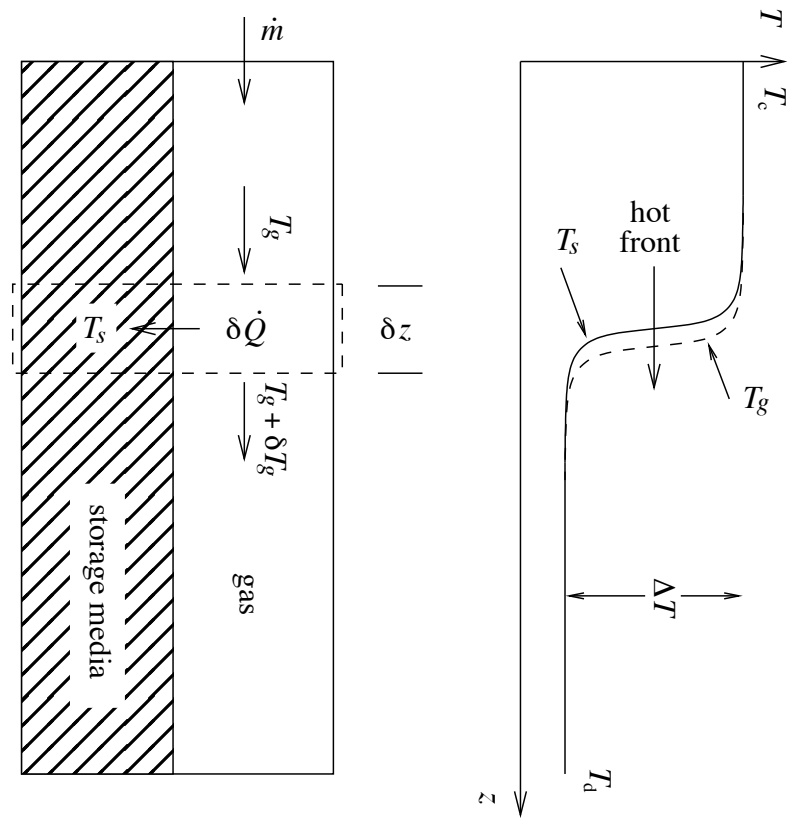

Figure 3: Schematic view of heat transfer process and typical axial temperature profiles for a hot reservoir during charge.

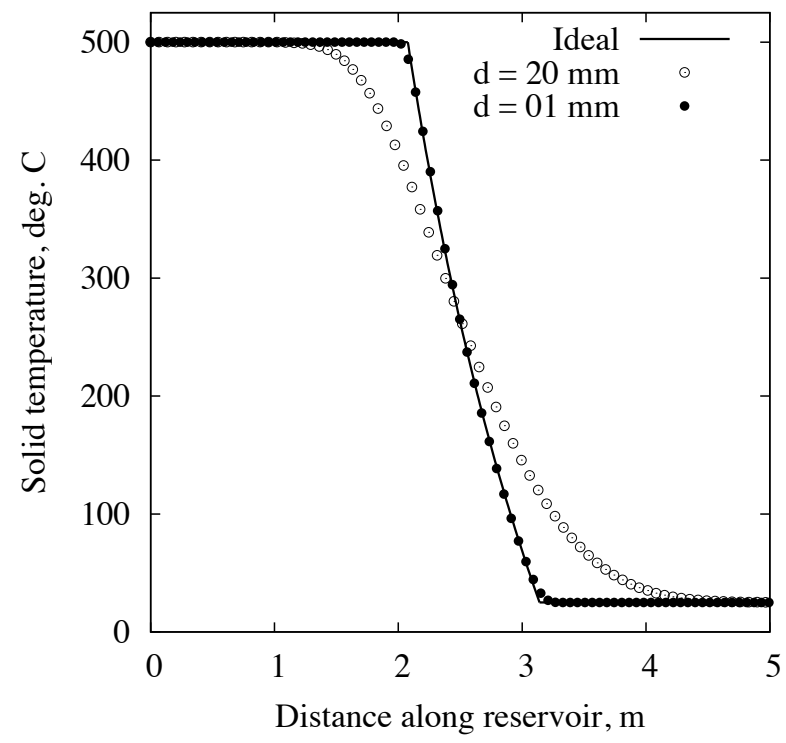

Figure 4: Solid temperature profiles for a hot reservoir during charge (geometry and other details are given in Appendix B). 


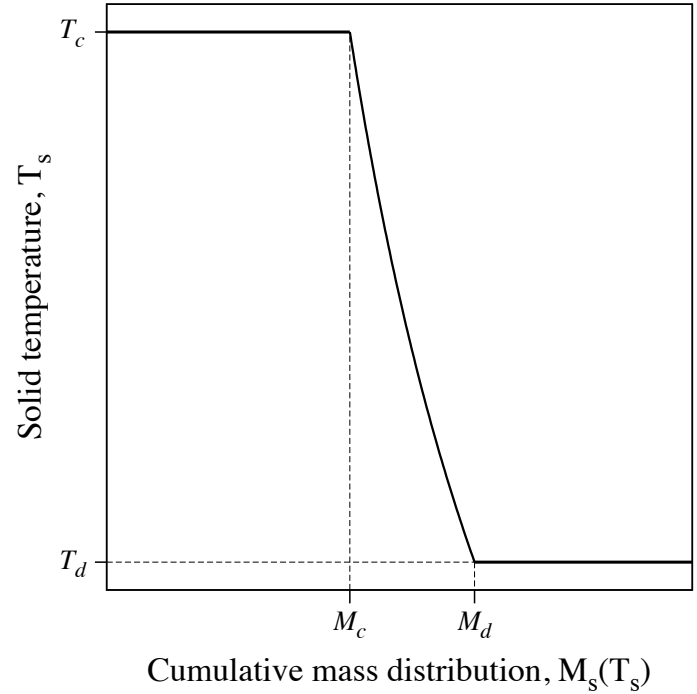

Figure 5: Sketch of the solid temperature profile for an ideal hot reservoir as a function of the cumulative mass at or above $T_{s}$.

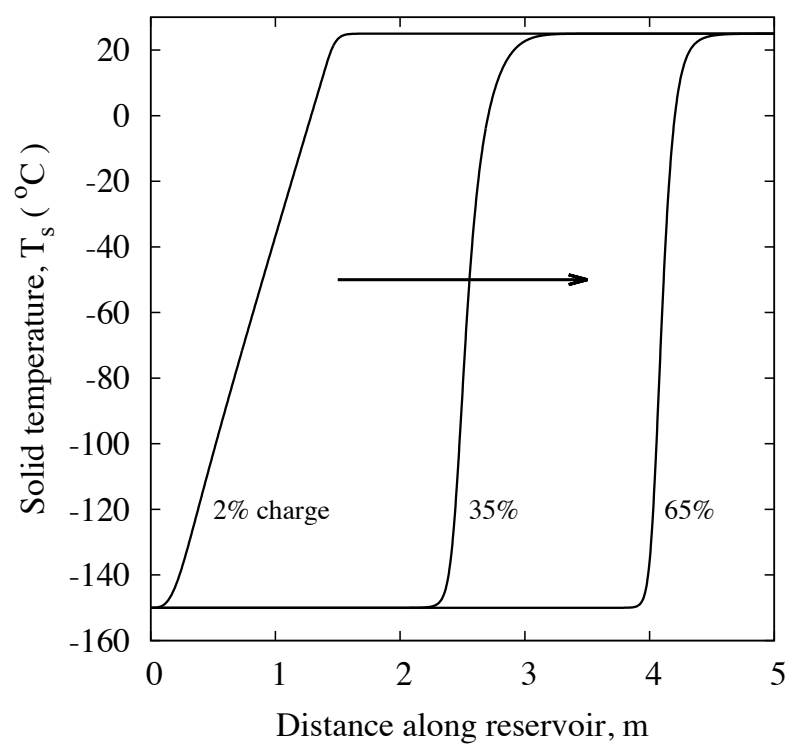

Figure 6: Numerical computation of wave 'catch-up' for a cold reservoir during charge. Geometry and other details are given in Appendix B. The storage material is $\mathrm{Fe}_{2} \mathrm{O}_{3}$. 


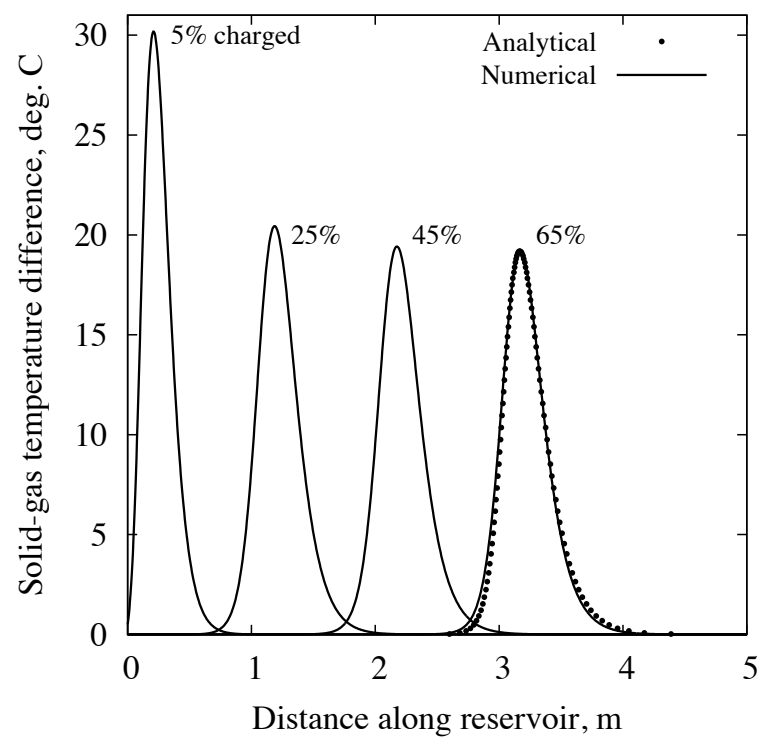

Figure 7: Solid-gas temperature difference profiles within a cold 'shock': comparison of the analytical result (eqs. 19 \& 20 ) with the numerical solutions. Geometry and other details are given in Appendix B. The solid is $\mathrm{Fe}_{2} \mathrm{O}_{3}$ with a linear fit of $c_{s}$ vs. $T_{s}$.

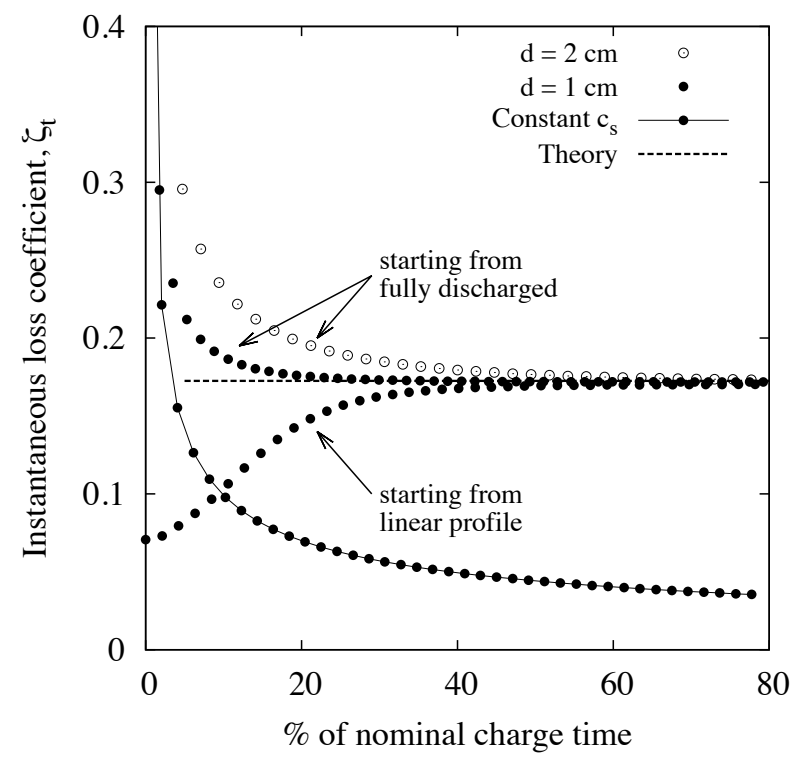

Figure 8: Evolution of the instantaneous loss coefficient, $\zeta_{t}$, for a cold reservoir undergoing charge. Geometry and other details are given in Appendix B. The material is $\mathrm{Fe}_{2} \mathrm{O}_{3}$. 

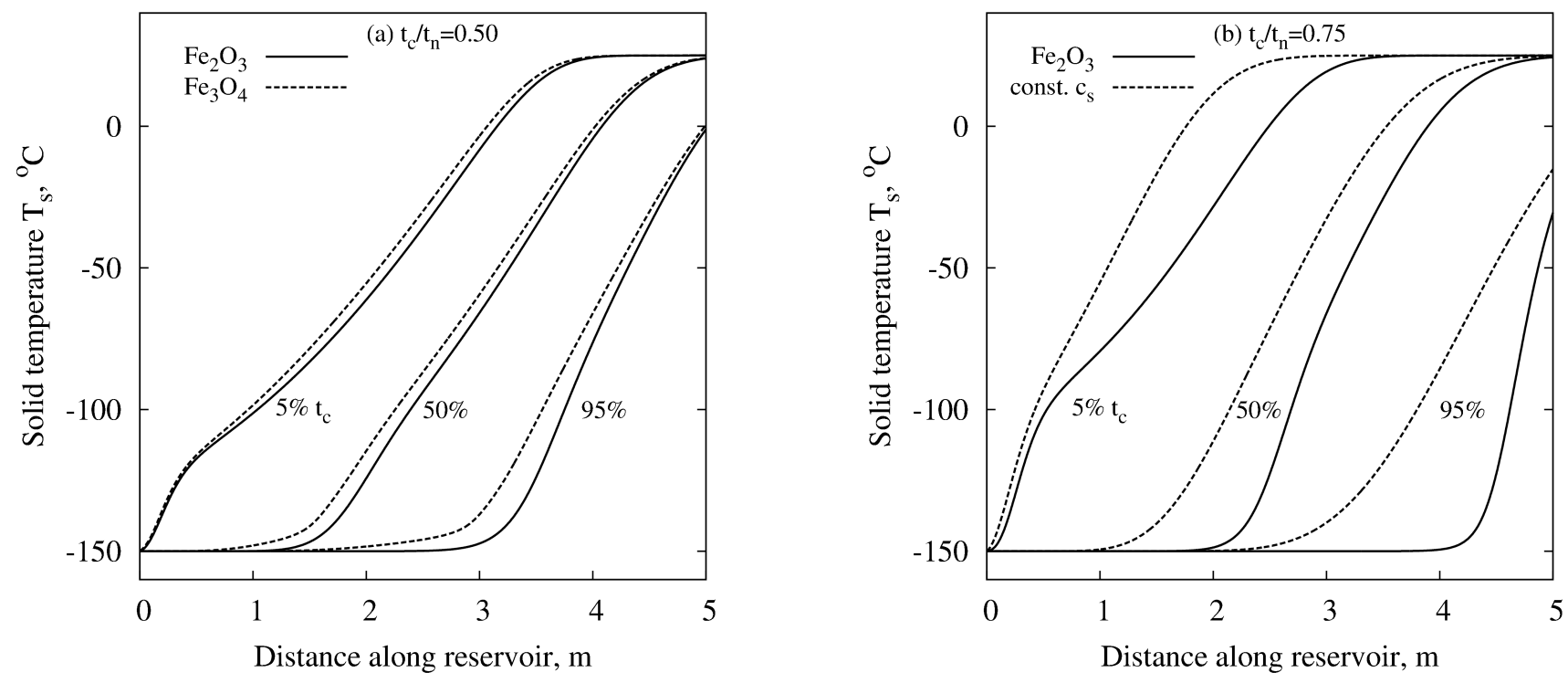

Figure 9: Temperature profiles in a cold reservoir under cyclic operation for two different charge periods, $t_{c}$. In each case profiles are shown 5\%,50\% and 95\% through the charge period. Charge and discharge periods are equal in each case.

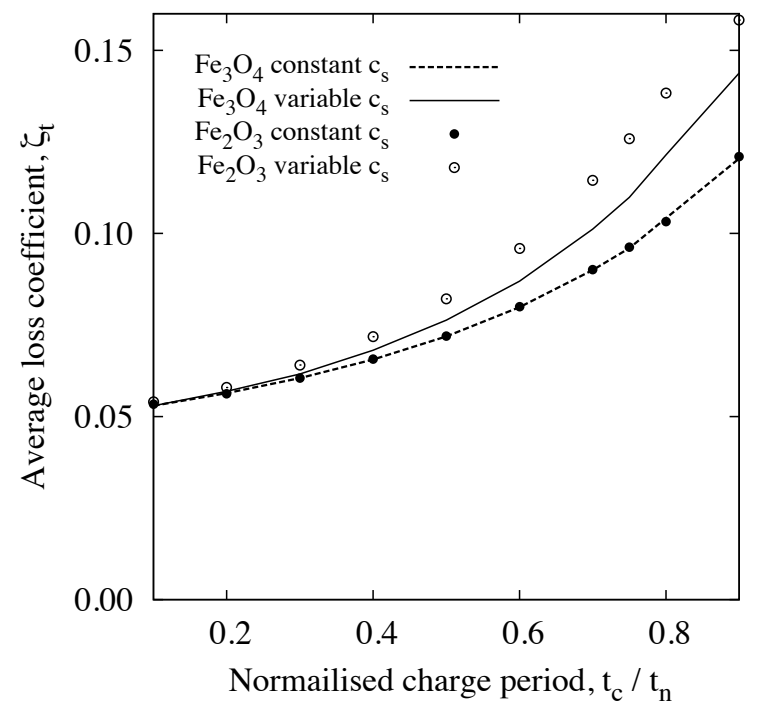

Figure 10: Thermodynamic loss for $20 \mathrm{~mm}$ diameter particles in a cold reservoir undergoing cyclic operation. 


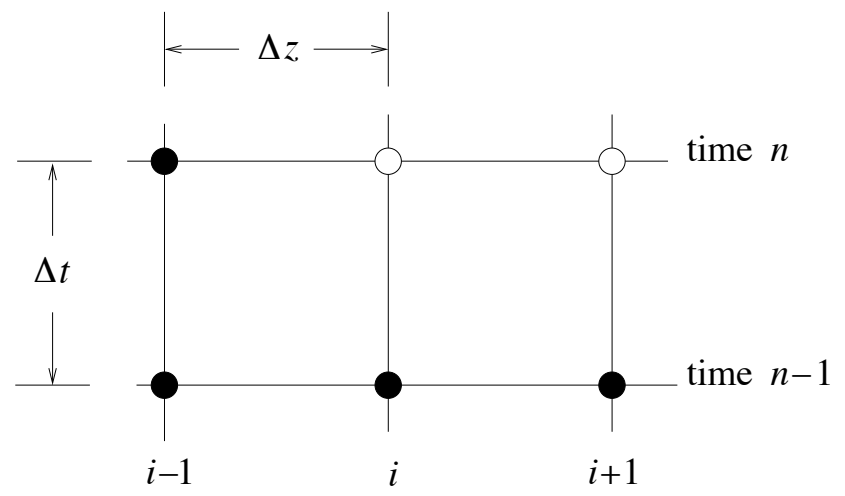

Figure 11: A section of the computation grid: temperatures are known at nodes marked $\bullet$ and unknown at nodes marked o. 